\title{
Poloidal asymmetric flow and current relaxation of ballooned transport during I-phase in ASDEX Upgrade
}

\author{
P. Manz ${ }^{1,2}$, G. Birkenmeier ${ }^{1,2}$, G. Fuchert ${ }^{2}$, M. Cavedon², G.D. Conway², M. Maraschek ${ }^{2}$, A. \\ Medvedeva $^{1,2}$ F. Mink ${ }^{2}$, B.D. Scott ${ }^{2}$, L.M. Shao ${ }^{2,3}$, U. Stroth ${ }^{2,1}$ and the ASDEX Upgrade Team \\ 1 Physik-Department E28, Technische Universität München, \\ James-Franck-Str. 1, 85748 Garching, Germany \\ 2 Max-Planck-Institut für Plasmaphysik, Boltzmannstr.2 ,85748 Garching, Germany \\ 3 Institute of Plasma Physics, Chinese Academy of Sciences, Hefei 230031, People's Republic of China
}

(Dated: May 12, 2016)

\begin{abstract}
Turbulence driven poloidal asymmetric parallel flow and current perturbations are studied for tokamak plasmas of circular geometry. Whereas zonal flows can lead to in-out asymmetry of parallel flows and currents via the Pfirsch-Schlüter mechanism, ballooned transport can result in an updown asymmetry due to the Stringer spin-up mechanism. Measurements of up-down asymmetric parallel current fluctuations occurring during the I-phase in ASDEX Upgrade are not responses to the equilibrium by the Pfirsch-Schlüter current, but can be interpreted as a response to strongly ballooned plasma transport coupled with the Stringer spin-up mechanism. A good agreement of the experimental measured limit-cycle frequencies during I-phase with the Stringer spin-up relaxation frequency is found.
\end{abstract}

PACS numbers:

\section{LIMIT-CYCLES AT THE L-H TRANSITION}

Limit-cycle oscillations (LCOs) preceding the transition from low (L-mode) to high confinement (H-mode) are intensively studied [1-9]. The regime dominated by LCOs is also called I-phase [1], dithering or transient mode. These limit-cycle oscillations have been predicted by a predator-prey model $[10,11]$, where turbulent generated flow and equilibrium flow shear are two competing predators interacting with drift-wave turbulence (prey). The experimental results differ strongly in detail. The turbulent generated flows appear as lowfrequency zonal flows $[2-9,12]$ or geodesic acoustic oscillations [1]. Whereas the basic mechanism of turbulence suppression by zonal flows [13-16], the existence of zonal flows during the $\mathrm{L}-\mathrm{H}$ transition [12] and the predatorprey dynamics in general [17] have been experimentally demonstrated, turbulence suppression by flow generation appears strong enough to trigger a transition into the $\mathrm{H}$ mode $[1,2,4,5,9,18]$ but some experiments show only weak or insufficient zonal flow activity at the L-H transition $[6,7,19,20]$. Recently also magnetic fluctuations are observed during these limit cycle oscillations [7, 8, 20-22] even though the predator-prey model $[10,11]$ is electrostatic in nature.

Here magnetic fluctuations during the I-phase in ASDEX Upgrade (AUG) are investigated. They show up as a very robust feature and occur during low [1] as well as medium densities $[20,22]$. In order to interpret the experiment, the evolution equations for these magnetic fluctuations are derived in the present contribution. Two turbulence driven mechanisms leading to a poloidal asymmetric magnetic signature are discussed. One is related to zonal flows, geodesic acoustic modes (GAMs) and the Pfirsch-Schlüter current, the other is related to ballooned transport and the Stringer spin-up.
Both mechanisms do not necessarily have to exclude each other. It will be shown that the magnetic fluctuations in AUG during I-phase can be caused by the relaxation current due to ballooned transport resembling the Stringer spin-up in principle. The experimental characteristics of the magnetic fluctuations during the I-phase in AUG is shown in the following chapter and subsequently indications of ballooned transport during I-phase in AUG are discussed. Afterward the governing equations are derived. From those the Stringer spin-up relaxation frequency is estimated and compared to the measured Iphase frequency in AUG. A good agreement with the experimental measurements is found.

\section{UP-DOWN ASYMMETRIC PARALLEL CURRENT FLUCTUATIONS DURING THE I-PHASE IN ASDEX UPGRADE}

Experiments have been carried out at the ASDEX Upgrade tokamak. The discharge shown exhibit a typical L- to H-transition with I-phase at line averaged density $\bar{n}_{e}=3 \cdot 10^{19} \mathrm{~m}^{3}$, toroidal field $B_{t}=2.3 \mathrm{~T}$, plasma current $I_{p}=0.8 \mathrm{MA}$, lower single null configuration, modulating electron cyclotron resonance heating power of $800 \mathrm{~kW}$. More details of this discharge can be found in Ref. [23]. Magnetic fluctuations measured by pick up coils sensitive to the poloidal magnetic component $\dot{B}_{\theta}$ appear during Iphase in ASDEX Upgrade [20, 22]. These fluctuations are strongly correlated with the flow represented by the Doppler shift from Doppler reflectometry [20, 22]. To obtain a global picture of the poloidal mode structure the cross-correlation between different poloidally displaced Mirnov coils on a poloidal cross-section has been calculated. During the I-phase in ASDEX Upgrade the magnetic fluctuations exhibit a pronounced up-down asym- 


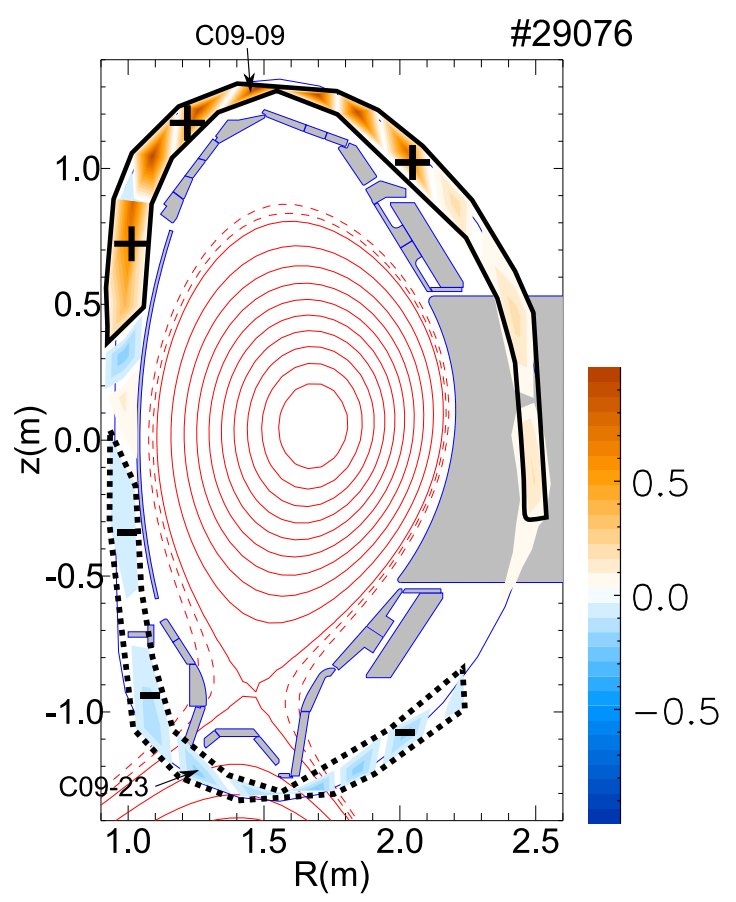

FIG. 1: Poloidal mode structure from cross-correlation of poloidally displaced Mirnov coils (C09-09 is the reference coil) at the same toroidal position.

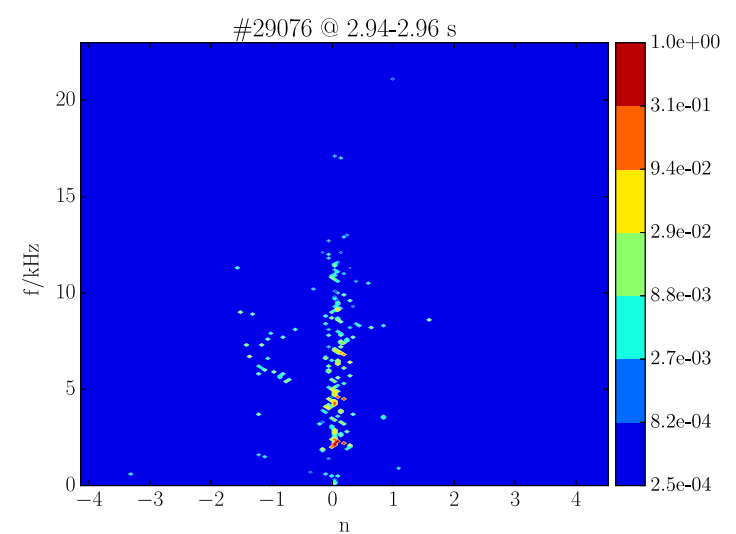

FIG. 2: Toroidal mode number at different frequencies during I-phase in ASDEX Upgrade.

metry as shown in Fig. 1. This corresponds to a poloidal mode number of $m=1$. For the measurement of the toroidal mode structure an array of poloidal field pickup coils, which is spread along the toroidal coordinate at fixed poloidal angle, was used (C09-17, C10-21, C0417, C05-21, C07-17). The poloidal position $\theta=-2.9$ rad is between inner midplane and inner divertor plate and therefore suitable to measure coherent fluctuations in the divertor region. The mode number is determined by the slope of a linear fit through the coil phases plotted over the coil positions, where the coil phase is given by $\varphi(f)=\arctan \left(\frac{\Im\left(\dot{\tilde{B}}_{\theta}(f)\right)}{\Re\left(\tilde{\tilde{B}}_{\theta}(f)\right)}\right)$, with the Fourier transformed coil signal $\dot{\tilde{B}}_{\theta}$. As seen in Fig. 2 the low frequency oscillations exhibit a dominant $n=0$ structure, where $n$ is the toroidal mode number. Therefore the magnetic structure is toroidally symmetric $(n=0)$ and up-down asymmetric $(m=1)$.

Similar magnetic oscillations have been recently observed in the I-phase of EAST [8] and HL-2A plasmas [7]. It has been proposed that these magnetic fluctuations are induced by the fluctuations of the equilibrium pressure gradient due to the equilibrium condition $\nabla p=\mathbf{J} \times \mathbf{B}$ $[7,8]$. However, such fluctuations would exhibit an in-out asymmetry as they are carried by the Pfirsch-Schlüter current $\left(\left\langle\tilde{J}_{\|} \cos s\right\rangle\right.$ with parallel current $\tilde{J}_{\|}$, ballooning angle $s$ and zonal average $\langle\cdot\rangle$ as described below). We explicitly do not exclude additional Pfirsch-Schlüter current perturbations. However, on AUG the up-down asymme$\operatorname{try}\left\langle\tilde{J}_{\|} \sin s\right\rangle$ is much more pronounced in amplitude than the in-out asymmetry [22]. Therefore, The focus of the present contribution is on the up-down asymmetric component. While the poloidal symmetry of the fluctuations has not been studied in HL-2A, the magnetic fluctuations in EAST show an up-down asymmetry instead of the expected in-out asymmetry of the Pfirsch-Schlüter current. This is consistent with the presented AUG results. As shown later these fluctuations can result as a response to the transport events during the I-phase and are not related to the Pfirsch-Schlüter balance of the equilibrium. The fluctuations are essentially the same as those in the M-mode observed in JET with 'unknown origin' [21].

\section{INDICATION OF ENHANCED BALLOONED TRANSPORT DURING I-PHASE IN ASDEX UPGRADE}

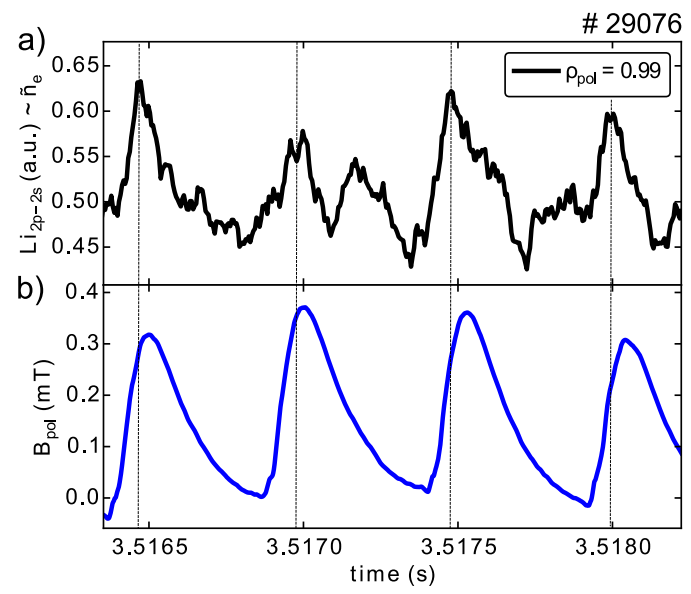

FIG. 3: a) Density measured with LiBES in the very edge of the confined region and b) poloidal magnetic field perturbations $B_{\theta}$ below the inner divertor.

The analytic investigation in the next chapter will reveal a coupling between up-down asymmetric current 


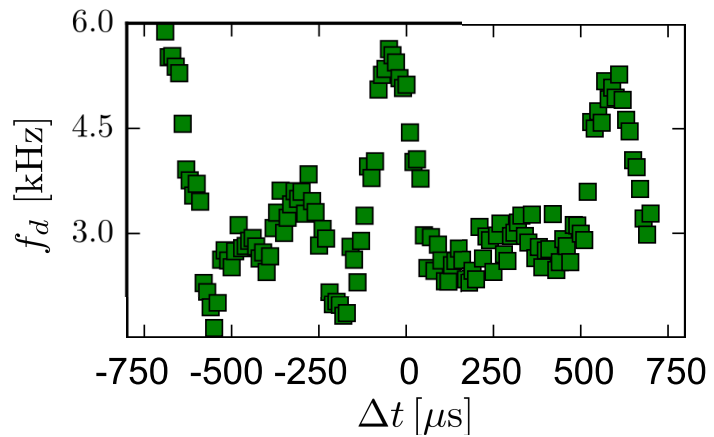

FIG. 4: Blob detection rate conditional averaged to the magnetic signal.

perturbations with in-out asymmetric (ballooned) transport in the confined region. Direct transport measurements are not available in the confined region. Hence, the survey of present experimental observations presented in the following is not sufficient to make a firm claim that ballooned transport is responsible for the observed magnetic signature explained above. However, a consistent behavior with this possibility is indicative.

First, the temporal correlation of density and magnetic fluctuations is discussed. In the confined region the density can be measured at the outboard midplane via lithium beam emission spectroscopy (LiBES) [24]. The magnetic signal $\dot{B}_{\theta}$ measured below the inner divertor target (C09-23) is correlated with the density perturbation measured in the very edge of the confined region $\left(\rho_{\text {pol }}=0.99\right)$ as shown in comparison in Fig. 3. Therefore, rises in the density induce an increase in the poloidal magnetic field. The parallel current associated with the poloidal magnetic field is directly proportional to magnetic field not to its temporal derivative. As the transport in the SOL increases as result of increasing transport in the confined region as shown in a previous study [22] this observation can be further supported by SOL measurements done with gas puff imaging (GPI). Blobs are associated with the interchange or resistive ballooning instability. Using a fast camera in AUG [25], the average blob detection rate was found to be a function of the phase of the magnetic fluctuations (measured below the inner divertor target (C09-23)). The blob detection rate is clearly correlated with the frequency of the magnetic fluctuations (Fig. 4). Furthermore, it seems that the detection rate of intermittent events peaks slightly prior to the magnetic fluctuations reach their maximum (Fig. 4). The appearance of blobs before the rise in the magnetic signal indicates that also the transport within the confined region increases before the magnetic signal. As both, LiBES and GPI diagnostics, measure at one poloidal position only, no conclusions can be made regarding the poloidal asymmetry.

Next, we want to discuss a possible general enhancement of ballooning. As prior to a burst in the poloidal magnetic field the transport from the confined region at the low-field side is not significantly reduced (it is actually enhanced as shown above), a reduction of density at the high-field side in the SOL would be a result of reduced transport from the confined region into the SOL at the high-field side directly. This would be an indication of increased ballooning at the separatrix. Indeed, previous measurements with the $\mathrm{X}$-point reciprocating probe in ASDEX Upgrade have shown that the onset of the pulsing in the I-phase is preceded by a fast $50 \%$ density drop at the high-field side X-point region [23] which lets suggest an increase of ballooning ( $\cos s$ contribution) of the transport prior to the L-I transition. Therefore, also the ballooning of the transport in the confined region should be enhanced in I-phase compared to L-mode.

In summary, poloidal magnetic perturbations as an indicator of the parallel current rise after an increase of density perturbations in the very edge of the confined region. Previous experimental results indicate an increase of ballooning of the transport in I-phase compared to L-mode [23].

\section{DYNAMICS OF UP-DOWN ASYMMETRIC PARALLEL CURRENTS AND FLOWS}

In this chapter the equations for the observed up-down asymmetric current perturbations will be derived based on the the Drift-ALFvén model DALF [26-28]. The basic equations are introduced in the next subsection. The experimentally observed poloidal asymmetric magnetic signature can be described by an $m=1, n=0$ mode which can be obtained by multiplying the observed physical quantity with the desired asymmetry $(\sin s$ or $\cos s$, respectively) in the ballooning angle and subsequently taking the flux surface average. This is called a sideband. The dynamics of zonal flows, geodesic acoustic modes and Pfirsch-Schlüter currents as done in Ref. [28] and the treatment of sidebands will be recapitulated in the subsection after that. The reader familiar with the DALF model may skip the first subsection and who is also familiar with Ref. [28] may skip the first two subsections. New aspects beyond the discussion in Ref. [28] start in the third subsection, where the general evolution equation for up-down asymmetric parallel current perturbations is given. Those perturbations are intrinsically related to up-down asymmetric parallel flows as well as in-out asymmetric pressure and vorticity (or perpendicular flow) perturbations. As the evolution equations of inout asymmetric pressure perturbations includes the ballooned transport the complete sideband balance recover the Stringer spin-up. Therefore, the third subsection is called the 'Stringer spin-up sideband balance'. In the last subsection the relaxation frequencies within the Stringer spin-up sideband balance are calculated and compared to the I-phase frequencies as measured in ASDEX Upgrade. 


\section{A. The drift-Alfvén model DALF}

The DALF model describes drift-Alfvén turbulence in toroidal geometry and therefore also includes interchange and MHD instabilities. It has been used mostly for numerical investigations of turbulence, however here only an analytical treatment is intended. It consists of evolution equations for the the vorticity $\tilde{\Omega}$

$$
\frac{d \tilde{\Omega}}{d t}=B \nabla_{\|} \frac{\tilde{J}_{\|}}{B}-\left(1+\tau_{i}\right) \mathcal{K}\left(\tilde{p_{e}}\right),
$$

the electron pressure $\tilde{p}_{e}$

$$
\frac{d \tilde{p}_{e}}{d t}+\tilde{v}_{E} \nabla p_{e}=B \nabla_{\|} \frac{\tilde{J}_{\|}-\tilde{u}_{\|}}{B}+\mathcal{K}\left(\tilde{\phi}-\tilde{p}_{e}\right),
$$

the parallel current $\tilde{J}_{\|}$

$$
\hat{\beta} \frac{\partial \tilde{A}_{\|}}{\partial t}+\mu \frac{d \tilde{J}_{\|}}{d t}=\nabla_{\|}\left(p_{e}+\tilde{p}_{e}-\tilde{\phi}\right)-C \tilde{J}_{\|} \cdot
$$

and the ion velocity

$$
\hat{\epsilon} \frac{d \tilde{u}_{\|}}{d t}=-\left(1+\tau_{i}\right) \nabla_{\|}\left(p_{e}+\tilde{p}_{e}\right)+\mu_{\|} \nabla_{\|}^{2} \tilde{u}_{\|} .
$$

The equations are completed by Ampere's law

$$
-\nabla_{\perp}^{2} \tilde{A}_{\|}=\tilde{J}_{\|}
$$

and polarization

$$
\frac{1}{B^{2}} \nabla_{\perp}^{2} \tilde{W}=\tilde{\Omega} .
$$

The normalization is done by the ion sound speed given by $c_{s}=\sqrt{T_{e} / m_{i}}$ and does not include the contribution of the ions, $\rho_{s}=\sqrt{T_{e} m_{i}} / e B$ with electron temperature $T_{e}$, ion mass $m_{i}$, magnetic field strength $B$ and elementary charge $e . d / d t=\partial / \partial t+v_{E} \cdot \nabla$ is the advective derivative with $E \times B$ velocity $v_{E}$. The main fluctuating quantities are the electrostatic potential $\tilde{\phi}=e \phi / T_{e 0}$ normalized to the background mean electron temperature $T_{e 0}$ and the electron pressure fluctuations $\tilde{p}_{e}=\tilde{p}_{e 1} / p_{e 0}$ normalized to a characteristic mean background pressure value $p_{e 0}$. The total ion flow stream function $\tilde{W}=\tilde{\phi}+\tau_{i} \tilde{p}_{e}$ determines the vorticity $\tilde{\Omega}=\left(1 / B^{2}\right) \nabla_{\perp}^{2} \tilde{W}$. The difference between parallel ion flow $\tilde{u}_{\|}$and current $\tilde{J}_{\|}$defines the parallel electron flow $\tilde{v}_{\|}=\tilde{u}_{\|}-\tilde{J}_{\|}$. Times are normalized to $L_{\perp} / c_{s}$, perpendicular spatial scales to $\rho_{s}$ and parallel to $q R$ with safety factor $q$ and major radius $R$. Here $L_{\perp}$ is the relevant gradient scale length, which is in most cases $L_{\perp}=-T_{e} / \nabla T_{e}$. For the pressure we consider the background together with the disturbance and drop the tilde symbol. In some cases we will investigate the total pressure $p=p_{e}+p_{i}$. Relative amplitudes of the fluctuations are given in $\rho_{s} / L_{\perp}$ which is the smallness parameter.

The coordinates used here are $(x, y, s)$, where $s$ is in direction of the unperturbed magnetic field line and $x$ and $y$ are radial and binormal, respectively, locally perpendicular to the unperturbed magnetic field line. A shifted metric coordinate system is used with a different $y$ coordinate at each location in $s$. It is important to note that in a tokamak the direction parallel to the magnetic field is not toroidal and perpendicular is not poloidal. In a flux tube coordinate system the parallel coordinate $s$ is represented by the poloidal angle $\theta$ defined in $[-\pi, \pi]$ with its origin at the outer midplane. The parallel wavenumber is given by $k_{\|}=\frac{m-n q}{q R}$ with poloidal mode number $m$ and toroidal mode number $n$, respectively. The local perpendicular or binormal wavenumber in drift direction is given by the toroidal wavenumber $k_{y}=\frac{n q}{r}$ with the radius of the flux tube $r$. The local shift, which is different at each radial position, allows to take the effect of magnetic shear into account. The curvature operator is given by $\mathcal{K}=\omega_{B}\left(\sin s \partial_{x}+\cos s \partial_{y}\right)$ with $\omega_{B}=2 L_{\perp} / R$ with the curvature radius $R$, which is set to the major radius. The first contribution to the curvature is the geodesic curvature the second the normal curvature. The flux surface (zonal) average is given by $\langle f\rangle=\oint \oint d s d y f$. The normalized magnetic field strength is $B=1$ with the component parallel to the field line $b_{s}=1$. The parallel derivative includes magnetic fluctuations of the fluxsurface $\nabla_{\|}=b_{s} \frac{\partial}{\partial s}+\tilde{b}_{x} \frac{\partial}{\partial x}+\tilde{b}_{y} \frac{\partial}{\partial y}$. They are calculated as $\tilde{b}_{x}=\hat{\beta} \frac{\partial \tilde{A}}{\partial y}$ and $\tilde{b}_{y}=-\hat{\beta} \frac{\partial \tilde{A}}{\partial x}$.

Different regimes are set by the square of the parallel/perpendicular scale ratio $\hat{\epsilon}=\left(q R / L_{\perp}\right)^{2}$, the normalized $\beta$ given by $\hat{\beta}=\left(4 \pi n T_{e} / B^{2}\right)\left(q R / L_{\perp}\right)^{2}$ and the normalized mass ratio $\hat{\mu}=\left(m_{e} / m_{i}\right)\left(q R / L_{\perp}\right)^{2}$, which determine the relative transit Alfvén and electron thermal frequencies, respectively. Amplitudes are normalized to $\rho_{s} / L_{\perp}$ with gradient scale length $L_{\perp}$. The parallel current is dissipated by collisions represented by $C=\hat{\mu}\left(0.51 \nu_{e} / c_{s} L_{\perp}\right)$ with collision frequency $\nu_{e}$. The ratio between ion and electron temperature is given by $\tau_{i}=T_{i} / T_{e}$. Flow damping is controlled by the neoclassical viscosity $\mu_{\|}$.

\section{B. Dynamics of zonal flows, geodesic acoustic modes and Pfirsch-Schlüter currents}

A detailed analysis of the energy transfer system between turbulence, zonal modes and further sidebands in an axis-symmetrical configuration is presented in Ref. [28]. Sidebands are modes with finite parallel wavevector $k_{\|} \neq 0$ but vanishing binormal wavevector $k_{y}=0$ (corresponding to $n=0, m \neq 0$ ). The sidebands are obtained by multiplying the evolution equations for current, flow, pressure and vorticity by the asymmetry function $\sin s$ or $\cos s$ first and take the zonal average subsequently. In this way the resulting modes exhibit a parallel wavenumber of $k_{\|}= \pm \frac{1}{q R}$ with poloidal and toroidal mode numbers of one and zero, respectively. Here $\langle\cdot\rangle$ denotes the flux surface average and $s$ is the ballooning angle [28]. As $s$ is defined to be zero at the outer midplane 
$\langle\sin s\rangle$ gives the up-down asymmetric components to the flux surface average and $\langle\cos s\rangle$ the in-out asymmetric component. The principal energetic flow is summarized in the following.

The zonal pressure evolution is

$$
\frac{\partial}{\partial t}\left\langle p_{e}\right\rangle=-\frac{\partial}{\partial x}\left\langle\tilde{v}_{E x} p_{e}+\tilde{b}_{x} \tilde{v}_{\|}\right\rangle-\omega_{B}\langle(p-\tilde{W}) \sin s\rangle .
$$

Through the gradient drive, energy is transferred from the background pressure $p_{0}$ to the turbulence $\tilde{p}$. This is done by the coupling between the second term on the left hand side of Eq. (2) with the first term on the right hand side of Eq. (7). Due to adiabatic coupling (first terms on the right hand side of Eqs. (1) and (2)) fluctuating energy is transferred to vorticity fluctuations $\tilde{\Omega}$. The zonal vorticity is obtained by taking the zonal average of Eq. (1)

$$
\frac{\partial}{\partial t}\langle\Omega\rangle=-\frac{\partial^{2}}{\partial x^{2}}\left\langle\tilde{v}_{E x} \tilde{u}_{y}\right\rangle+\frac{1}{\hat{\beta}} \frac{\partial^{2}}{\partial x^{2}}\left\langle\tilde{b}_{x} \tilde{b}_{y}\right\rangle-\omega_{B} \frac{\partial}{\partial x}\langle p \sin s\rangle
$$

giving the zonal flow $\left\langle\tilde{u}_{y}\right\rangle=\left\langle\frac{\partial \tilde{W}}{\partial x}\right\rangle$

$$
\frac{\partial}{\partial t}\left\langle\tilde{u}_{y}\right\rangle=-\frac{\partial}{\partial x}\left\langle\tilde{v}_{E x} \tilde{u}_{y}\right\rangle+\frac{1}{\hat{\beta}} \frac{\partial}{\partial x}\left\langle\tilde{b}_{x} \tilde{b}_{y}\right\rangle-\omega_{B}\langle p \sin s\rangle .
$$

Note the zonal flow includes the ion diamagnetic contribution and $\left\langle\frac{\partial \tilde{u}_{y}}{\partial x}\right\rangle=\langle\tilde{\Omega}\rangle$. The turbulent $\tilde{\Omega}$ drives the zonal vorticity $\langle\Omega\rangle=\langle\tilde{\Omega}\rangle$ via the Reynolds stress $\frac{\partial}{\partial x}\left\langle\tilde{v}_{E x} \tilde{u}_{y}\right\rangle$ (Eq. (9)). The zonal vorticity determines the zonal flow. The second term is the Maxwell stress. The zonal flow is saturated by driving to an up-down asymmetric pressure sideband $\langle\tilde{p} \sin s\rangle$. This results from the geodesic curvature $\omega_{B} \sin s$. The evolution of this pressure sideband is

$$
\begin{aligned}
\frac{\partial}{\partial t}\left\langle p_{e} \sin s\right\rangle= & -\frac{\partial}{\partial x}\left\langle\left(\tilde{v}_{E x} p_{e}+\tilde{b}_{x} \tilde{v}_{\|}\right) \sin s\right\rangle+\left\langle v_{\|} \cos s\right\rangle \\
& -\frac{\omega_{B}}{2}\left\langle\left(\frac{\partial p}{\partial x}-\tilde{u}_{y}\right)(1-\cos 2 s)\right\rangle \cdot(10)
\end{aligned}
$$

The coupling to the zonal flow is within the last term. The oscillation between the zonal flow $\left\langle\tilde{u}_{y}\right\rangle$ and this pressure sideband $\left\langle p_{e} \sin s\right\rangle$ is the geodesic acoustic mode. Neglecting all other contributions that $\frac{\partial}{\partial t}\left\langle p_{e} \sin s\right\rangle=$ $\frac{1}{2} \omega_{B}\left\langle\tilde{u}_{y}\right\rangle$ and also just considering the coupling to this pressure sideband for the zonal flow equation (9) $\frac{\partial}{\partial t}\left\langle\tilde{u}_{y}\right\rangle=-\left(1+\tau_{i}\right) \omega_{B}\left\langle p_{e} \sin s\right\rangle$ a natural frequency of $\omega_{B} \sqrt{\left(1+\tau_{i}\right) / 2}$ or in physical units $f_{G A M}=\sqrt{2} c_{s i} / 2 \pi R$ with $c_{s i}^{2}=\left(1+\tau_{i}\right) T_{e} / m_{i}$ can be derived which is the GAM frequency. This pressure sideband can also be driven by an up-down asymmetric transport as seen at the first term on the right hand side of Eq. (10). Most damping occurs due to $\left\langle\tilde{v}_{\|} \cos s\right\rangle$ which is connected to the flow and current by $\left\langle\tilde{v}_{\|} \cos s\right\rangle=\left\langle\tilde{u}_{\|} \cos s\right\rangle-\left\langle\tilde{J}_{\|} \cos s\right\rangle$. Via adiabatic coupling the energy is transferred from the pressure sideband to perturbations in the Pfirsch-Schlüter current
$\left\langle\tilde{J}_{\|} \cos s\right\rangle$ where the energy is dissipated by the collisionality

$$
\begin{aligned}
\frac{\partial}{\partial t}\left\langle\left(\hat{\beta} \tilde{A}_{\|}+\hat{\mu} \tilde{J}_{\|}\right) \cos s\right\rangle= & -\frac{\partial}{\partial x}\left\langle\tilde{v}_{E x} \hat{\mu} \tilde{J}_{\|} \cos s\right\rangle \\
& +\left\langle\tilde{b}_{x}\left(\frac{\partial p}{\partial x}-\frac{\partial \tilde{\phi}}{\partial x}\right) \cos s\right\rangle \\
& +\langle(p-\tilde{W}) \sin s\rangle \\
& -C\left\langle\tilde{J}_{\|} \cos s\right\rangle
\end{aligned}
$$

As shown in a previous numerical investigation [28] the most important sidebands related to zonal flow physics are the up-down asymmetric pressure sideband $\langle p \sin s\rangle$ taking part in the geodesic acoustic oscillation (GAM), the vorticity sideband $\langle\tilde{\Omega} \sin s\rangle$ (directly linked to the sidebands of the ion stream function $\langle\tilde{W} \sin s\rangle$ and flow $\left.\left\langle\tilde{u}_{y} \sin s\right\rangle\right)$ as well as the Pfirsch-Schlüter current $\left\langle\tilde{J}_{\|} \cos s\right\rangle$. The Pfirsch-Schlüter sound sideband $\left\langle\tilde{u}_{\|} \cos s\right\rangle$ plays a minor role.

\section{Stringer-Spin up sideband balance}

The experimentally observed up-down asymmetry $(\sin s)$ in the parallel current $\tilde{J}_{\|}$as described in the second section can be written as $\left\langle\tilde{J}_{\|} \sin s\right\rangle$. The evolution can be obtained from Eq. (3) multiplied with $\sin s$ for the up-down asymmetry and zonally averaged afterward

$$
\begin{aligned}
\frac{\partial}{\partial t}\left\langle\left(\hat{\beta} \tilde{A}_{\|}+\hat{\mu} \tilde{J}_{\|}\right) \sin s\right\rangle= & -\frac{\partial}{\partial x}\left\langle\hat{\mu} \tilde{v}_{E x} \tilde{J}_{\|} \sin s\right\rangle \\
& +\left\langle\tilde{b}_{x}\left(\frac{\partial \tilde{p}}{\partial x}-\frac{\partial \phi}{\partial x}\right) \sin s\right\rangle \\
& -\langle(p-\tilde{W}) \cos s\rangle \\
& -C\left\langle\tilde{J}_{\|} \sin s\right\rangle .
\end{aligned}
$$

Here, $\left\langle\sin s \nabla_{\|} p\right\rangle=\left\langle\nabla_{\|}(p \sin s)\right\rangle-\left\langle p \nabla_{\|} \sin s\right\rangle=$ $-\langle p \cos s\rangle$ is used since the zonal average of parallel derivatives vanishes. Up-down asymmetric current perturbations are coupled to the in-out asymmetric pressure and vorticity sidebands via $\langle(p-\tilde{W}) \cos s\rangle$ which will be investigated later. Due to $\left\langle\tilde{u}_{\|} \sin s\right\rangle=\left\langle\tilde{v}_{\|} \sin s\right\rangle+$ $\left\langle\tilde{J}_{\|} \sin s\right\rangle$ up-down asymmetric current perturbations are coupled to up-down asymmetric flow perturbations which are investigated next. The up-down asymmetry in parallel momentum evolves according to $(\langle\mathrm{Eq} .(4) \sin s\rangle)$

$$
\begin{aligned}
\hat{\epsilon} \frac{\partial}{\partial t}\left\langle\tilde{u}_{\|} \sin s\right\rangle= & -\frac{\partial}{\partial x}\left\langle\hat{\epsilon} \tilde{v}_{E x} \tilde{u}_{\|} \sin s\right\rangle \\
& -\left\langle\tilde{b}_{x} \frac{\partial \tilde{p}}{\partial x} \sin s\right\rangle \\
& +\langle p \cos s\rangle-\mu_{\|}\left\langle\tilde{u}_{\|} \sin s\right\rangle .
\end{aligned}
$$

Up-down asymmetric flows are driven by the up-down asymmetric radial-parallel Reynolds stress $\left\langle\hat{\epsilon} \tilde{v}_{E x} u_{\|} \sin s\right\rangle$ and are saturated by the parallel ion viscosity $\mu_{\|}$. The up-down asymmetric radial-parallel Reynolds stress is 
the up-down asymmetric parallel momentum transport and is large as the symmetry of the gyrokinetic distribution function $f\left(s, v_{\|}\right)=f\left(-s,-v_{\|}\right)$gives an updown asymmetric statistical moment of parallel momentum and due to ballooning of the potential the parallel momentum transport is up-down asymmetric [29]. This asymmetry is also the reason for the need of parallel symmetry breaking for intrinsic rotation, which is not needed to generate an up-down asymmetric flow. Additionally we find a coupling with the in-out asymmetry in the pressure, whose evolution is given by $(\langle$ Eq. $(2) \cos s\rangle)$

$$
\begin{aligned}
\frac{\partial}{\partial t}\left\langle p_{e} \cos s\right\rangle= & -\frac{\partial}{\partial x}\left\langle\tilde{v}_{E x} p_{e} \cos s\right\rangle \\
& -\frac{\partial}{\partial x}\left\langle\tilde{b}_{x} \tilde{v}_{\|} \cos s\right\rangle+\left\langle\tilde{J}_{\|} \sin s\right\rangle-\left\langle\tilde{u}_{\|} \sin s\right\rangle \\
& -\frac{\omega_{B}}{2}\left\langle\left(\frac{\partial p}{\partial x}-\tilde{u}_{y}\right) \sin 2 s\right\rangle
\end{aligned}
$$

The in-out asymmetry in the pressure can be understood as an in-out movement of the plasma column or by a local flattening of the pressure profile on the low field side for example. It can result from ballooned transport $\left\langle\tilde{v}_{E x} p_{e} \cos s\right\rangle$, an up-down asymmetric parallel electron flow $\left\langle\tilde{v}_{\|} \sin s\right\rangle$, which is connected to the parallel ion flow and current by $\left\langle\tilde{v}_{\|} \sin s\right\rangle=\left\langle\tilde{u}_{\|} \sin s\right\rangle-\left\langle\tilde{J}_{\|} \sin s\right\rangle$. In particular the ballooned transport $\left\langle\tilde{v}_{E x} p_{e} \cos s\right\rangle$ can be expected to be quite large and could provide the most important turbulent drive for the here described sideband balance. Elongation of the magnetic equilibrium introduces $m=2$ modes [30,31], which appear to drive inout asymmetric pressure disturbance by $\left\langle\left(\frac{\partial p}{\partial x}-\tilde{u}_{y}\right) \sin 2 s\right\rangle$ which contains coupling to the pressure gradient $\partial p / \partial x$ as well as to the perpendicular flow $\tilde{u}_{y}=\partial \tilde{W} / \partial x$ with ion stream function $\tilde{W}$. The term arises from the curvature $\langle\cos s \mathcal{K}(\tilde{\phi}-p)\rangle=\left\langle\cos s \sin s \omega_{B} \frac{\partial}{\partial x}(\tilde{\phi}-p)\right\rangle$ with the geodesic contribution to the curvature $\mathcal{K}=\omega_{B} \sin s \frac{\partial}{\partial x}$. Now $\cos s \sin s=\frac{1}{2} \sin 2 s$ induces the coupling to the second harmonic. In general, a disturbance of the axissymmetry of the magnetic equilibrium can drive inout asymmetric pressure perturbations as well as in-out asymmetric flow perturbations.

The evolution of this sideband system is closed by the in-out asymmetric vorticity $\langle\tilde{\Omega} \cos s\rangle=\left\langle\nabla_{\perp}^{2} \tilde{W} \cos s\right\rangle$

$$
\begin{aligned}
\frac{\partial}{\partial t}\langle\tilde{\Omega} \cos s\rangle= & -\frac{\partial^{2}}{\partial x^{2}}\left\langle\tilde{v}_{E x} \tilde{u}_{y} \cos s\right\rangle \\
& +\frac{\partial^{2}}{\partial x^{2}} \frac{1}{\hat{\beta}}\left\langle\tilde{b}_{x} \tilde{b}_{y} \cos s\right\rangle \\
& +\left\langle\tilde{J}_{\|} \sin s\right\rangle-\frac{\omega_{B}}{2}\left\langle\frac{\partial p}{\partial x} \sin 2 s\right\rangle .
\end{aligned}
$$

Besides the disturbance of the axis-symmetry of the equilibrium, it is coupled to the ballooned perpendicular Reynolds stress and again the up-down asymmetry in the parallel current. Therefore also in an up-down symmetric equilibrium a ballooned perpendicular Reynolds stress leads to up-down asymmetric parallel current perturbations $=\left\langle\tilde{J}_{\|} \sin s\right\rangle=\frac{\partial^{2}}{\partial x^{2}}\left\langle\tilde{v}_{E x} \tilde{u}_{y} \cos s\right\rangle$. Due to the coupling of the in-out asymmetry in the pressure and flow, up-down asymmetric parallel current perturbations can be expected.

In conclusion, the observed up-down asymmetric magnetic fluctuations $\left\langle\tilde{J}_{\|} \sin s\right\rangle$ are coupled to up-down asymmetric flow perturbations $\left\langle\tilde{u}_{\|} \sin s\right\rangle$ via $\left\langle\tilde{u}_{\|} \sin s\right\rangle=$ $\left\langle\tilde{v}_{\|} \sin s\right\rangle+\left\langle\tilde{J}_{\|} \sin s\right\rangle$ and in-out symmetric pressure $\langle p \cos s\rangle$ via Eqs. (12) and (14). Furthermore it is coupled to vorticity (as well as binormal shear flow) $\langle\tilde{\Omega} \sin s\rangle$ perturbations via Eqs. (12) and (15). Ballooned transport and Reynolds stress as well as the up-down asymmetric parallel-radial Reynolds stress are involved in the sideband balance. All these quantities cannot be expected to be small a priori. The induction of an up-down asymmetric parallel flow $\left\langle\tilde{u}_{\|} \sin s\right\rangle$ by the ballooned transport $\left\langle\tilde{v}_{E x} p_{e} \cos s\right\rangle$ is known since a long time. This is the Stringer spin-up [32, 33].

\section{Relaxation frequencies of poloidal asymmetric transport}

The presented set of equations (12)-(15) allows for multiple limit-cycles scenarios. The aim is to study the basic relaxation processes similar to the relaxation process in the Pfirsch-Schlüter sideband balance resulting in the geodesic acoustic mode. The first and second terms on the r.h.s. of Eqs. (12)-(15) are the electrostatic and electromagnetic transport terms. These are nonlinear and vanish with linearization. The last terms in Eqs. (14) and (15) are geometric corrections which can be expected to be small in circular geometry. Of course the inclusion of all terms gives a more precise result. Two examples of relaxation processes leading to limit cycles are presented. As a first example the set is reduced to the here observed phenomena in ASDEX Upgrade: the enhanced ballooned transport included in the in-out asymmetry in the pressure Eq. (14) and up-down asymmetric poloidal magnetic field fluctuations corresponding to up-down asymmetric parallel current fluctuations Eq. (12). All other terms are neglected. Linearizing Eqs. (14) and (12) in the electrostatic limit yields $i \omega_{J P}\left\langle p_{e} \cos s\right\rangle=\left\langle\tilde{J}_{\|} \sin s\right\rangle$ and $i \hat{\mu} \omega_{J P}\left\langle\tilde{J}_{\|} \sin s\right\rangle=-\left(1+\tau_{i}\right)\left\langle p_{e} \cos s\right\rangle$. This exhibits an eigenfrequency of $\omega_{J P}=\sqrt{\left(1+\tau_{i}\right) / \hat{\mu}}$ (renormalization gives $\left.f_{J P}=\sqrt{m_{i} / m_{e}}\left(c_{s i} /(2 \pi q R)\right)\right)$ in the few hundred $\mathrm{kHz}$ range for ASDEX Upgrade parameters. These frequencies are too high compared to the common limitcycle oscillation in the I-phase of about a few $\mathrm{kHz}$. However, they might be related to the high frequency limit cycles observed in H-mode plasmas in EAST accompanied by strong intermittent transport [34].

As a second example the ion relaxation of the ballooned transport is investigated. Replacing the parallel electron Eq. (12) by that of the ions (13) gives a coupling of the ballooned transport with the up-down asymmetric 


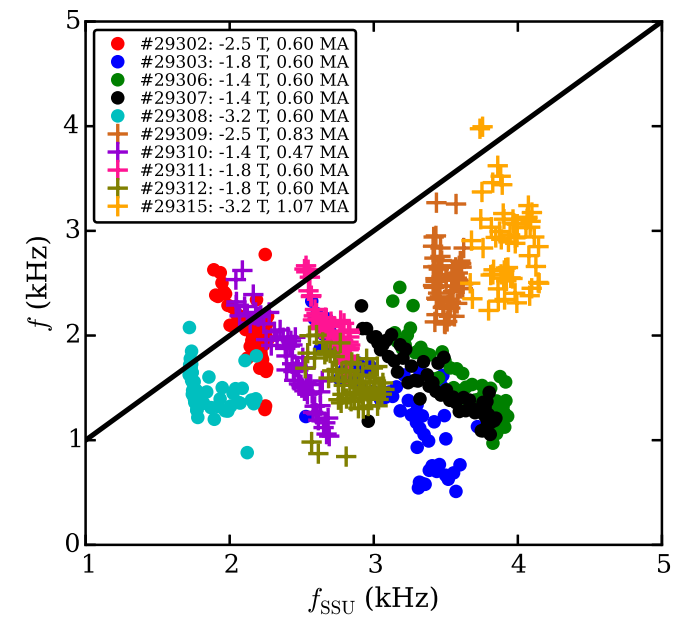

FIG. 5: Stringer spin-up relaxation frequency $f_{S S U}=$ $c_{s} /(2 \pi q R)$ against Limit-cycle frequencies of several AUG discharges.

flow, which is the Stringer spin-up $[32,33]$. Linearizing both equations gives $i \omega_{S S U}\left\langle\tilde{u}_{\|} \sin s\right\rangle=\frac{\left(1+\tau_{i}\right)}{\hat{\epsilon}}\left\langle p_{e} \cos s\right\rangle$ and $i \omega_{S S U}\left\langle p_{e} \cos s\right\rangle=-\left\langle\tilde{u}_{\|} \sin s\right\rangle$ corresponding to an eigenfrequency of $\omega_{S S U}=\sqrt{\left(1+\tau_{i}\right) / \hat{\epsilon}}$ (renormalization gives $\left.f_{S S U}=c_{s i} /(2 \pi q R)\right)$. Note that this scaling is by a factor $1 /(\sqrt{2} q)$ smaller than the GAM frequency. This scaling exhibits a scaling $f_{S S U} \sim 1 / q \sim I_{p}$ similar to that observed in the M-mode in JET [21]. It cannot explain the result of the multivariate linear regression for $\mathrm{AUG}$ limit cycles which gives $f_{I-\text { phase }} \sim 1 / \beta_{t, p e d} q_{95}^{3 / 2}$ with the toroidal plasma beta $\beta_{t, \text { ped }}$ measured in the pedestal [22]. Still, a comparison with the recent data base of I-phase discharges [22] shows an overall good qualitative agreement (Fig. 5). The experimental frequencies are below the frequency predicted by the Stringer spin-up relaxation frequencies. It seems this relaxation gives an upper boundary for the I-phase frequency. The inclusion of any kind of damping in the equation system would reduce $f_{S S U}$. However, considering the derivation of $f_{S S U}$ is done for a simple large aspect ratio circular plasma. The corresponding GAM frequency of $f_{G A M}=\sqrt{2} c_{s i} / 2 \pi R$ also predicts smaller frequencies as actually measured in the edge in AUG [35]. Taken divertor shaped equilibria may substantially improve the agreement between experiment and theory.

Limit cycle oscillations can arise from every energy transfer term within the system. Also for the Stringer spin up a Lotka-Volterra-type set of equations can be obtained

$\frac{\partial}{\partial t} \frac{1}{2}\left\langle p_{e} \cos s\right\rangle^{2}=\left\langle p_{e} \cos s\right\rangle \frac{\partial}{\partial x}\left\langle\tilde{v}_{E x} \tilde{p} \cos \right\rangle-\left\langle p_{e} \cos s\right\rangle\left\langle\tilde{u}_{\|} \sin s\right\rangle$, $\hat{\epsilon} \frac{\partial}{\partial t} \frac{1}{2}\left\langle\tilde{u}_{\|} \sin s\right\rangle^{2}=+\left(1+\tau_{i}\right)\left\langle p_{e} \cos s\right\rangle\left\langle\tilde{u}_{\|} \sin s\right\rangle-\mu\left\langle\tilde{u}_{\|} \sin s\right\rangle^{2}$

which is driven by ballooned transport, damped by neoclassical flow damping and coupled by the energy exchange term $\langle p \cos s\rangle\left\langle\tilde{u}_{\|} \sin s\right\rangle$ which can lead to limit cycle oscillations. Here, the in-out asymmetry in the pressure takes the role of the prey and the up-down asymmetric flow perturbation (the Stringer spin up) takes the role of the predator. Compared to Volterra's original model, here the predator-prey interaction constitutes a root term. Root terms in population dynamics can for example result from herd behavior of the prey [36]. The dynamic properties are essentially alike, while the modifications allow for explicit solutions [37]. The frequency of the limit cycles is given by the prefactors of the predator-prey interaction terms and not by the drive and damping terms [37], it coincides with the eigenfrequency above $\omega_{S S U}=\sqrt{\left(1+\tau_{i}\right) / \hat{\epsilon}}$ determined by the parallel/perpendicular scale ratio $\hat{\epsilon}=\left(q R / L_{\perp}\right)^{2}$.

\section{DISCUSSION AND CONCLUSION}

The magnetic signature of fluctuations in the I-phase or limit-cycle regime of ASDEX Upgrade has been investigated. Up-down asymmetric parallel current fluctuations are observed. These fluctuations can result as a response to the plasma transport. A preceding fast density drop in the high-field side X-point region [23] points to an increase of ballooning at the separatrix prior to the transition to I-phase. During I-phase the poloidal magnetic field perturbations follow density perturbations in the very edge of the confined region measured via lithium beam emission spectroscopy [22].

The dynamics of up-down asymmetric currents and flows have been derived based on the model of Scott [28] describing also the interaction between electromagnetic $E \times B$ turbulence, zonal flows, geodesic acoustic modes and perturbations in the Pfirsch-Schlüter current. Updown asymmetric flows and parallel currents are intrinsically coupled to in-out asymmetric pressure and vorticity perturbations which depicts an extended Stringer spin-up sideband balance. They are not related to the Pfirsch-Schlüter current and to the equilibrium pressure evolution.

The observation of limit-cycles is not necessarily related to zonal flows or Reynolds stresses and it is not necessarily related to critical gradient models. The Stringer spin-up sideband balance provides several relaxation phenomena, which all can be described by limit-cycle processes likewise. The eigenfrequencies of two of them have been calculated. A comparison with recent I-phase discharges in ASEDX Upgrade [22] shows a good agreement of the experimental measured limit-cycle frequencies with the Stringer spin-up relaxation frequency. Even though the Stringer spin-up relaxation frequency can induce limit-cycles it should be noted that the Stringer 
spin-up sideband balance does not include terms with direct impact on the zonal dynamics as the transport, the Reynolds stress or the pressure gradient themselves. It describes their distribution on a flux surface. Even though the Stringer spin-up relaxation frequency is therefore much likely not the cause of the limit-cycles in the I-phase, as both frequencies are close together resonance phenomena can be expected. Furthermore, the terms in the Stringer spin-up sideband balance can be used for diagnostics purpose. In strongly ballooned turbulence it can be used to measure the transport or Reynolds stress indirectly by magnetic fluctuations. The derived Springer spin-up sideband balance is not restricted to the I-phase and should be important in situations where the transport is strongly ballooned as for example during ELMs or for kinetic ballooning turbulence.

\section{Acknowledgements}

This work has been carried out within the framework of the EUROfusion Consortium and has received funding from the Euratom research and training programme 2014-2018 under grant agreement No 633053. The views and opinions expressed herein do not necessarily reflect those of the European Commission.
[1] G. D. Conway, C. Angioni, F. Ryter, P. Sauter, and J. Vicente, Phys. Rev. Lett. 106, 065001 (2011).

[2] L. Schmitz, L. Zeng, T. L. Rhodes, J. C. Hillesheim, E. J. Doyle, R. J. Groebner, W. A. Peebles, K. H. Burrell, and G. Wang, Phys. Rev. Lett. 108, 155002 (2012).

[3] G. S. Xu, B. N. Wan, H. Q. Wang, H. Y. Guo, H. L. Zhao, A. D. Liu, V. Naulin, P. H. Diamond, G. R. Tynan, M. Xu, et al., Phys. Rev. Lett. 107, 125001 (2011).

[4] P. Manz, G. S. Xu, B. N. Wan, H. Q. Wang, H. Y. Guo, I. Cziegler, N. Fedorczak, C. Holland, S. H. Müller, S. C. Thakur, et al., Phys. Plasmas 19, 072311 (2012).

[5] G. R. Tynan, M. Xu, P. H. Diamond, J. A. Boedo, I. Cziegler, N. Fedorczak, P. Manz, K. Miki, S. Thakur, L. Schmitz, et al., Nucl. Fusion 53, 073053 (2013).

[6] T. Kobayashi, K. Itoh, T. Ido, K. Kamiya, S.-I. Itoh, Y. Miura, Y. Nagashima, A. Fujisawa, S. Inagaki, K. Ida, et al., Phys. Rev. Lett. 111, 035002 (2013).

[7] J. Cheng et al., Phys. Rev. Lett. 110, 265002 (2013).

[8] G. S. Xu, H. Q. Wang, M. Xu, B. N. Wan, H. Y. Guo, P. H. Diamond, G. R. Tynan, R. Chen, N. Yan, D. F. Kong, et al., Nuclear Fusion 54, 103002 (2014).

[9] Z. Yan, G. R. McKee, R. Fonck, P. Gohil, R. J. Groebner, and T. H. Osborne, Phys. Rev. Lett. 112, 125002 (2014).

[10] P. H. Diamond, Y. M. Liang, B. A. Carreras, and P. W. Terry, Phys. Rev. Lett. 72, 2565 (1994).

[11] E. Kim and P. H. Diamond, Phys. Rev. Lett. 90, 185006 (2003).

[12] J. C. Hillesheim, E. Delabie, H. Meyer, C. F. Maggi, L. Meneses, E. Poli, and JET Contributors, Phys. Rev. Lett. 116, 065002 (2016).

[13] P. Manz, M. Ramisch, and U. Stroth, Phys. Rev. Lett. 103, 165004 (2009).

[14] M. Xu, G. R. Tynan, P. H. Diamond, S. H. Müller, C. Holland, J. H. Yu, and Z. Yan, Phys. Rev. Lett. 107, 055003 (2011).

[15] M. Xu, G. R. Tynan, P. Diamond, P. Manz, C. Holland, N. Fedorczak, S. Chakraborty Thakur, J. H. Yu, K. J. Zhao, J. Q. Dong, et al., Phys. Rev. Lett. 108, 245001 (2012).

[16] N. Fedorczak, P. Manz, S. Chakraborty Thakur, M. Xu, and G. R. Tynan, Plasma Phys. \& Controlled Fusion 55, 025011 (2013).

[17] P. Manz, M. Ramisch, and U. Stroth, Phys. Rev. E 82, 056403 (2010).
[18] I. Cziegler, G. R. Tynan, P. H. Diamond, A. E. Hubbard, J. W. Hughes, J. Irby, and J. L. Terry, Nucl. Fusion 55, 0083007 (2015).

[19] K. Itoh, T. Kobayashi, T. Ido, S.-I. Itoh, and K. Kamiya, Plasma Phys. \& Controlled Fusion 57, 092001 (2015).

[20] M. Cavedon, T. Puetterich, E. Viezzer, G. Birkenmeier, T. Happel, F. M. Laggner, P. Manz, F. Ryter, U. Stroth, and ASDEX Upgrade Team, Nucl. Fusion, submitted (2016).

[21] E. R. Solano et al., Proc. of the $40^{\text {th }}$ Europ. Conf. on Plasma Physics, Espoo, Finland p. P4.111 (2013).

[22] G. Birkenmeier, M. Cavedon, G. D. Conway, P. Manz, G. Fuchert, F. M. Laggner, T. Happel, A. Medvedeva, V. Nikolaeva, D. Prisiazhniuk, L. M. Shao, M. Maraschek, T. Pütterich, F. Ryter, M. Willensdorfer, E. Wolfrum, U. Stroth, H. Zohm, the ASDEX Upgrade Team, Nucl. Fusion, submitted (2016).

[23] S. H. Müller, G. D. Conway, G. Birkenmeier, D. Carralero, T. Happel, A. Herrmann, P. Manz, P. de Marne, A. Mlynek, H. W. Müller, et al., Phys. Plasmas 21, 042301 (2014).

[24] M. Willensdorfer, G. Birkenmeier, R. Fischer, F. M. Laggner, E. Wolfrum, G. Veres, F. Aumayr, D. Carralero, L. Guimaris, B. Kurzan, et al., Plasma Phys. \& Controlled Fusion 56, 025008 (2014).

[25] G. Fuchert, G. Birkenmeier, D. Carralero, T. Lunt, P. Manz, H. W. Müller, B. Nold, M. Ramisch, V. Rohde, U. Stroth, et al., Plasma Phys. \& Controlled Fusion 56, 125001 (2014).

[26] B. D. Scott, Contrib. Plas. Phys. 38, 171 (1998).

[27] B. Scott, Plasma Phys. \& Controlled Fusion 40, 823 (1998).

[28] B. D. Scott, New J. Phys. 7, 92 (2005).

[29] Y. Camenen, A. G. Peeters, C. Angioni, F. J. Casson, W. A. Hornsby, A. P. Snodin, and D. Strintzi, Phys. Plasmas 16, 062501 (2009).

[30] J. Ball, F. I. Parra, M. Barnes, W. Dorland, G. W. Hammett, P. Rodrigues, and N. F. Loureiro, Plasma Phys. \& Controlled Fusion 56, 095014 (2014).

[31] T. Watari, Y. Hamada, A. Fujisawa, K. Toi, and K. Itoh, Phys. Plasmas 12, 062304 (2005).

[32] T. E. Stringer, Phys. Rev. Lett. 22, 770 (1969).

[33] A. B. Hassam and J. F. Drake, Phys. Fluids B: Plasma Phys. 5, 4022 (1993). 
[34] G. S. Xu, H. Q. Wang, B. N. Wan, H. Y. Guo, V. Naulin, P. H. Diamond, G. R. Tynan, M. Xu, N. Yan, W. Zhang, et al., Phys. Plasmas 19, 122502 (2012).

[35] G. D. Conway, C. Tröster, B. Scott, K. Hallatschek, and the ASDEX Upgrade Team, Plasma Phys. \& Controlled Fusion 50, 055009 (2008).
[36] S. N. Matia and S. Alam, Univ. J. Appl. Math. 1, 251 (2013).

[37] M. Arrigoni and A. Steiner, Acta Biotheoretica 32, 123 (1983). 